\title{
A more thorough analysis of water rockets: Moist adiabats, transient flows, and inertial forces in a soda bottle
}

\author{
Cedric J. Gommes \\ Department of Chemical Engineering, University of Liège, B6A Allée du 6 août, 3, B-4000 Liège, Belgium
}

(Received 19 May 2009; accepted 12 October 2009)

\begin{abstract}
Although water rockets are widely used to illustrate first year physics principles, accurate measurements show that they outperform the usual textbook analysis at the beginning of the thrust phase. This paper gives a more thorough analysis of this problem. It is shown that the air expansion in the rocket is accompanied by water vapor condensation, which provides an extra thrust; the downward acceleration of water within the rocket also contributes to the thrust, an effect that is negligible in other types of rockets; the apparent gravity resulting from the acceleration of the rocket contributes as much to water ejection as does the pressure difference between the inside and outside of the rocket; and the water flow is transient, which precludes the use of Bernoulli's equation. Although none of these effects is negligible, they mostly cancel each other, and the overall accuracy of the analysis is only marginally improved. There remains a difference between theory and experiment with water rockets. (C) 2010 American Association of Physics Teachers.

[DOI: $10.1119 / 1.3257702]$
\end{abstract}

\section{INTRODUCTION}

The water rocket ${ }^{1}$ is a popular toy that is often used in first year physics courses to illustrate Newton's laws of motion and rocket propulsion. In its simplest version, a water rocket is made of a soda bottle, a bicycle pump, a rubber stopper, and some piping (see Fig. 1). The bottle is half-filled with water, turned upside-down, and air is pushed inside the bottle via a flexible pipe that runs through the stopper. When the pressure builds up, the stopper eventually pops out of the neck. The water is then ejected and the rocket takes off. Witnesses of the launch of a water rocket cannot but be amazed that such a simple device can reach a height of tens of meters in a fraction of a second.

The popularity of water rockets extends beyond physics classrooms, with many existing associations and competitions organized worldwide. ${ }^{1}$ The more than 5000 videos posted on YouTube with the words "water rocket" in their title testify to their popularity. Some of these videos involve elaborate technical developments such as multistage water rockets, nozzles that adapt to the pressure, the replacement of water by foam or flour, underwater rocket launches, and even a water-propelled human flight. The public's passionate explorations with water rockets contrast with the small number of articles devoted to their analysis. I found only two papers $^{2,3}$ that treat the simplest possible rocket, similar to that shown in Fig. 1.

In the cited papers, the air expansion in the rocket is modeled as an isothermal ${ }^{3}$ or adiabatic ${ }^{2}$ process involving dry air, which enables the authors to estimate the pressure at any stage of water ejection. Based on Bernoulli's steady state equation, the pressure is then converted to a water ejection velocity at the nozzle, from which the thrust is estimated via the classical equation of rocket propulsion. Finally, Newton's laws of motion are solved numerically to predict the flight of the rocket. This analysis enables the time of flight to be predicted within the experimental uncertainties of a first year laboratory project. $^{3}$ However, more accurate measurements with a high-speed camera show that the acceleration of the rocket is substantially underestimated at the beginning of the thrust phase. ${ }^{2}$

Simple observations of water rockets hint at physical phe- nomena that were not considered in Refs. 2 and 3. First, the rocket is filled with fog at the end of a launch, as is visible in the inset of Fig. 2(b). Water condensation is an exothermic process that is expected to contribute positively to the thrust. Second, the acceleration of the rocket is huge, which suggests that the noninertial contribution to the apparent gravity in the rocket might help significantly water ejection. The rocket in Fig. 2 reaches a height of about $4 \mathrm{~m}$ in $0.2 \mathrm{~s}$, which implies an average acceleration of $200 \mathrm{~m} / \mathrm{s}^{2}$. A more accurate measurement ${ }^{2}$ with a high-speed camera leads to an acceleration of $100 \mathrm{~g}$ 's. The flow of water is rapid not just at the nozzle but also inside the rocket. The cloud in Fig. 2(b) forms at the end of ejection and appears after less than $0.2 \mathrm{~s}$. A more accurate ${ }^{2}$ ejection time is $\tau=0.1 \mathrm{~s}$. Because the height of water in the rocket is initially $H=10 \mathrm{~cm}$, this time converts to a velocity of $U=1 \mathrm{~m} / \mathrm{s}$ inside the rocket. The latter motion carries a momentum that may also contribute positively to the thrust.

In this paper an analysis of the water rocket is proposed, which takes account of the phenomena we have mentioned. A detailed physical analysis is presented in Sec. II, and the governing equations are derived. In Sec. III numerical solutions are compared with published experimental data. ${ }^{2}$

\section{PHYSICAL ANALYSIS}

\section{A. Moist air expansion}

Air expansion is the only source of energy of the rocket. As mentioned in Sec. I, it is accompanied by vapor condensation. The specific issue addressed here is the relation between the pressure $P$ and the volume $V$ of the expanding moist air. This relation determines the total amount of energy released during the ejection.

The first question is whether the expansion is adiabatic ${ }^{2}$ or isothermal as some authors claim. ${ }^{3}$ For the process to be isothermal, heat would have to diffuse into the rocket from outside to keep the temperature constant. Generally, during a time $\tau$, heat diffuses in air over a distance $\delta_{h}$ given by ${ }^{4,5}$ 


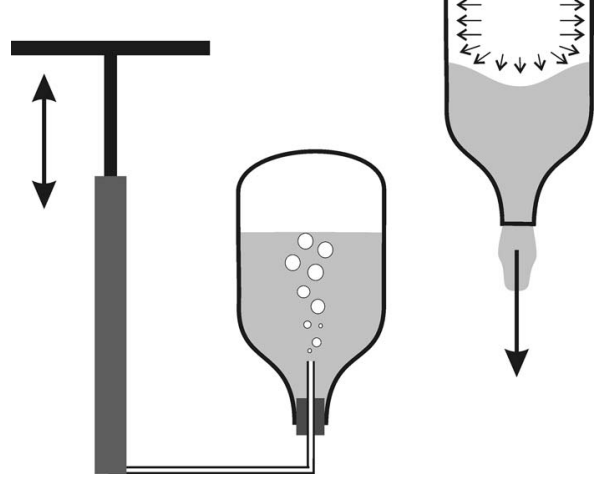

Fig. 1. In its simplest version, a water rocket is made of a soda bottle partially filled with water, in which air is injected with a bicycle pump. When the pressure increases, the stopper eventually pops out, water is ejected, and the rocket takes off.

$$
\delta_{h}^{2} \approx \chi \tau,
$$

where $\chi$ is the heat diffusivity of air. If we use the value $\chi=0.014 \mathrm{~cm}^{2} / \mathrm{s}$ and $\tau=0.1 \mathrm{~s}$, we find $\delta_{h}=0.04 \mathrm{~cm}$. Because this distance is much smaller than the radius of the rocket, the gas expansion has to be modeled as an adiabatic process.

Adiabaticity does not necessarily imply that $P V^{\gamma}$ is a constant, with $\gamma=1.4$ for diatomic gases. ${ }^{6,7}$ The cooling of air during an adiabatic expansion induces the condensation of the water vapor it contains. Because condensation is exothermic, the temperature does not drop as rapidly as if the air was dry, which keeps the pressure higher than predicted by the $P V^{\gamma}$ relation. Neglecting this effect leads to an underestimation of the work performed by the expanding air, and hence of the thrust.

The pressure-volume relation is derived, assuming that the total entropy resulting from dry air, water vapor, and condensed water (the fog) is constant during the adiabatic expansion. Assuming that air is an ideal gas, its molar entropy $s_{a}$ is written as ${ }^{6,8}$

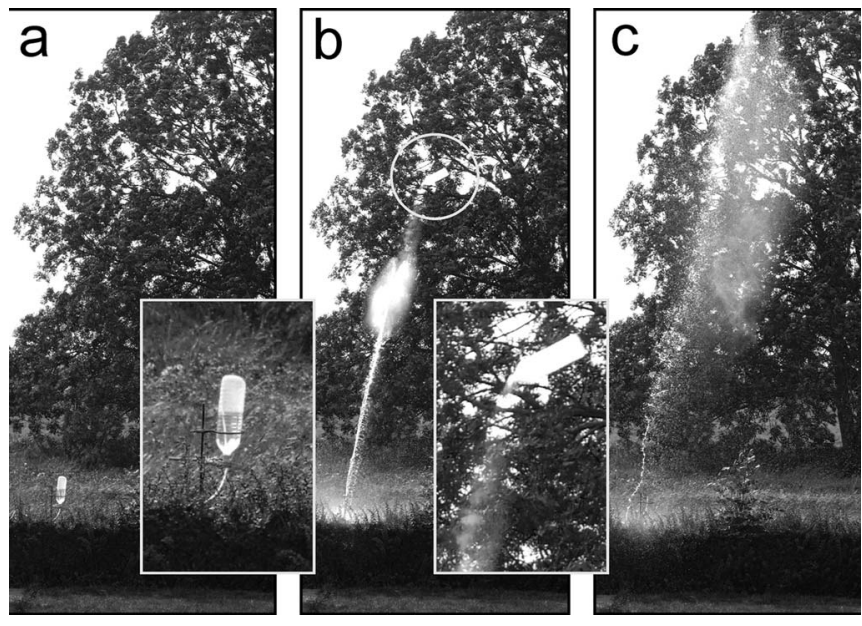

Fig. 2. Snapshots taken at times (a) $t=0 \mathrm{~s}$, (b) $t=0.2 \mathrm{~s}$, and $t=0.4 \mathrm{~s}$ during the launch of a water rocket. The insets show the rocket at the onset of ejection and soon after the end of ejection. Note that the ejected water in the cloud of (b) is moving upward.

$$
s_{a}(T, V)=s_{a}\left(T_{0}, V_{0}\right)+c_{V}^{a} \ln \left[\left(\frac{T}{T_{0}}\right)\left(\frac{V}{V_{0}}\right)^{\gamma-1}\right],
$$

where $c_{V}^{a}$ is the specific heat of air and $V$ is the volume. The 0 subscript refers to any arbitrarily chosen reference state. The molar entropy of water vapor in equilibrium with liquid water at temperature $T$ is written as ${ }^{6,8}$

$$
s_{V}(T)=s_{l}(T)+\frac{\Delta h_{V}}{T},
$$

where $s_{l}(T)$ is the molar entropy of the liquid water and $\Delta h_{V}$ is the molar enthalpy of vaporization of water. The molar entropy of liquid water $s_{l}(T)$ is ${ }^{6}$

$$
s_{l}(T)=s_{l}\left(T_{0}\right)+c_{p}^{l} \ln \left(\frac{T}{T_{0}}\right),
$$

where the specific heat of water $c_{P}^{l}$ has been assumed to be temperature independent.

The number of molecules in each of the three phases-air, water vapor, and liquid water-is obtained by assuming thermodynamic equilibrium. This assumption implies that the partial pressure of water vapor at any stage of the expansion is equal to the saturating pressure $P_{V}(T)$. By using the ideal gas law, $P_{V}(T)$ is converted to the total number of vapor molecules $N_{V}$ in the volume $V$. The total number of water molecules $N_{L}$ in the liquid is the difference between the initial and the current values of $N_{V}$. The constant number of air molecules in the rocket $N_{a}$ is determined as a function of the initial pressure $P_{i}$, volume $V_{i}$, and temperature $T_{i}$ by subtracting the contribution of water vapor to the total pressure.

The temperature-volume relation during the adiabatic expansion of moist air is obtained by conserving the total entropy $S=N_{a} s_{a}+N_{V} s_{v}+N_{c} s_{l}$ during the adiabatic expansion. We use Eqs. (2)-(4) to find

$$
\begin{aligned}
& {[1-}\left.\frac{P_{V}\left(T_{i}\right)}{P_{i}}\right] c_{V} \ln \left(\left(\frac{T}{T_{i}}\right)\left(\frac{V}{V_{i}}\right)^{\gamma-1}\right)+\left(\frac{T_{i}}{T}\right) \\
& \quad \times\left(\frac{V}{V_{i}}\right) \frac{P_{V}(T)}{P_{i}} \frac{\Delta h_{V}}{T}+\frac{P_{V}\left(T_{i}\right)}{P_{i}}\left(c_{p}^{l} \ln \left(\frac{T}{T_{i}}\right)-\frac{\Delta h_{V}}{T_{i}}\right)=0 .
\end{aligned}
$$

For the case of dry air, that is, for $P_{V}(T)=P_{V}\left(T_{i}\right)=0$, the solution of Eq. (5) is $T V^{(\gamma-1)}=$ constant, as expected. For moist air, however, Eq. (5) has to be solved to estimate the temperature $T$ reached after an adiabatic expansion. Knowing that temperature, the corresponding pressure is obtained by the ideal gas law.

Solutions of Eq. (5) are plotted in Fig. 3(a) for three values of $P_{i}$ and three values of $T_{i}$. The dry adiabats $\left(P V^{\gamma}\right.$ $=$ constant $)$ and isotherms $(P V=$ constant $)$ are shown for comparison. The numerical values used for the calculation are $c_{V}=20 \mathrm{~J} / \mathrm{mol} \mathrm{K},{ }^{8} c_{P}^{l}=75.3 \mathrm{~J} / \mathrm{mol} \mathrm{K},{ }^{6} \Delta h_{V}=45051 \mathrm{~J} / \mathrm{mol},{ }^{8}$ and $\gamma=1.4$; the saturating pressure was estimated from the Clausius-Clapeyron equation, ${ }^{6-8}$ with $P_{V}\left(T_{0}\right)=1$ bar at $T_{0}$ $=100{ }^{\circ} \mathrm{C}$.

The moist adiabats in Fig. 3(a) are found to be well approximated by a polytropic process ${ }^{8}$ of the type $P V^{\beta}$ $=$ constant (dotted lines). The exponent $\beta$ obtained by a leastsquares fit is found to depend mostly on the initial relative humidity $P_{V}\left(T_{i}\right) / P_{i}$, as shown in Fig. 3(b). The dependency is well described by the empirical function 

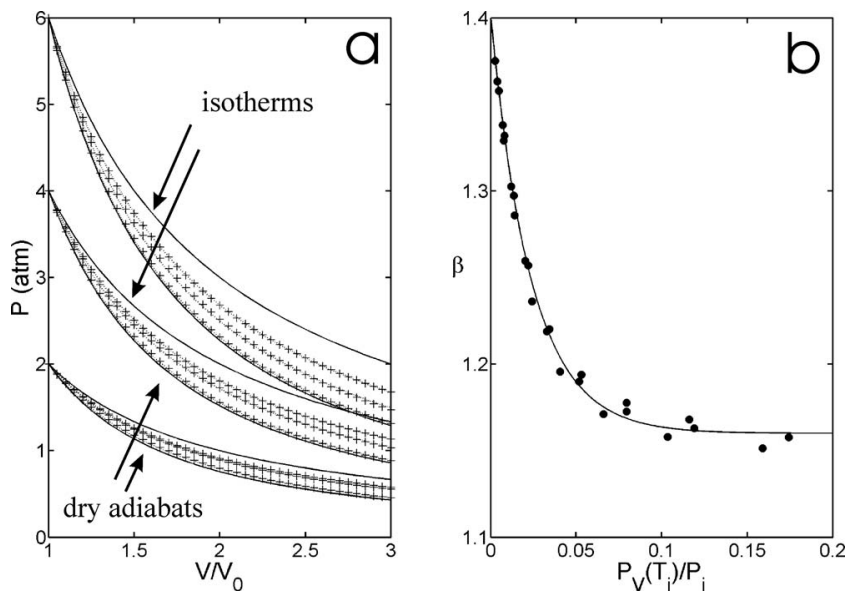

Fig. 3. (a) Pressure-volume curves of moist air during adiabatic expansion, starting from $P_{i}=2,4$, and 6 bars, and initially in equilibrium with water at $T_{i}=10,50$, and $90{ }^{\circ} \mathrm{C}(+)$. The solid lines are isotherms ( $p V=$ constant) and dry adiabats ( $p V^{\gamma}=$ constant). (b) Moist adiabats are approximated by a polytropic process of the form $p V^{\beta}=$ constant [dotted lines in (a)], the exponent of which is a function of the initial relative humidity.

$$
\beta=1.15+(1.4-1.15) \exp \left(-36 \frac{P_{V}\left(T_{i}\right)}{P_{i}}\right),
$$

which reduces to $\beta=\gamma=1.4$ for dry air.

Figure 4 shows a plot of the amount of work performed by a given volume of moist air, initially pressurized at $P_{i}=3,4$, and 7 bars, when it adiabatically doubles its volume against atmospheric pressure. In Fig. 4, the work is normalized to the amount of work performed by dry air in the same conditions. From Fig. 4, we see that air compressed to 3 bars performs $20 \%$ more work when it is saturated with water at $70{ }^{\circ} \mathrm{C}$ than when it is dry. In the experiments of Ref. 2 with $P_{i}$ $=3.4$ bars and $P_{i}=6.8$ bars, and $T_{i}=25^{\circ} \mathrm{C}$, the corresponding exponents are estimated to be $\beta=1.31$ and $\beta=1.35$. In both cases, the extra work resulting from water condensation is expected to be only a few percent. The same conclusion applies to the launches reported in Ref. 3 with $P_{i}=4$ bars.

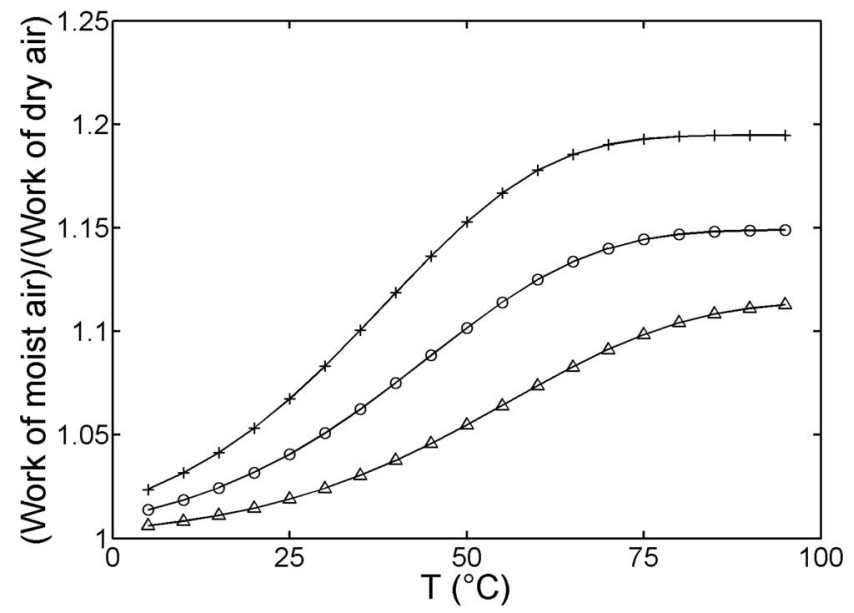

Fig. 4. Mechanical work performed by moist air upon doubling adiabatically its volume against atmospheric pressure, as a function of the initial temperature $T_{i}$ and initial pressure $P_{i}=3$ bars $(+), 4$ bars $(\bigcirc)$, and 7 bars $(\triangle)$. The work is normalized by the work performed by dry air under the same conditions.

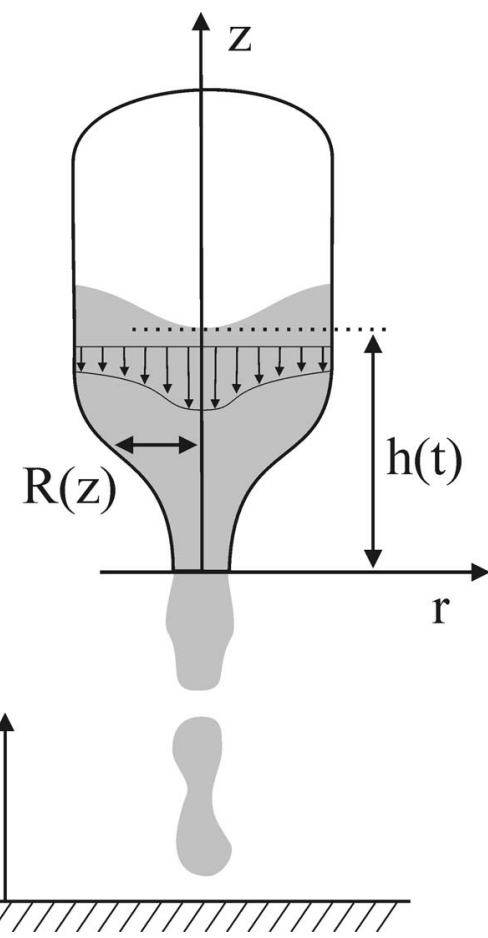

Fig. 5. The two reference frames used in the present analysis, and the meaning of some symbols. The free surface of water does not remain flat if the velocity profile is not uniform.

\section{B. Water ejection}

The determination of the rate of water ejection is a problem in fluid mechanics. The starting point of the analysis is to assess the importance of viscous forces. Using reasoning similar to Eq. (1), the distance $\delta_{v}$ over which momentum diffuses in a fluid during a time $\tau$ due to its viscosity is ${ }^{4,5}$

$$
\delta_{V}^{2} \approx v \tau
$$

where $\nu$ is the kinematic viscosity of the liquid. For water ${ }^{4}$ at $20{ }^{\circ} \mathrm{C}, \quad \nu=0.01 \mathrm{~cm}^{2} / \mathrm{s}$. Using $\tau=0.1 \mathrm{~s}$ leads to $\delta_{v}$ $=0.3 \mathrm{~mm}$. This small value means that the flow of water out of the rocket is not affected by viscosity, except for a submillimeter thin boundary layer. Water can therefore be assumed to be inviscid in this context, and ejection can be analyzed with Euler's equation.

The general form of Euler's equation is ${ }^{4,5}$

$$
\frac{\partial \vec{u}}{\partial t}+(\vec{u} \cdot \nabla) \vec{u}=-\frac{1}{\rho} \nabla P+\vec{g},
$$

where $\vec{u}$ is the vector velocity of water, $P$ is the pressure, $\rho$ is the density, and $\vec{g}$ is the gravitational field; $\vec{u}$ and $P$ are generally space and time dependent. When Eq. (8) is integrated along the axis of the rocket, from the nozzle up to the free surface of the liquid (see Fig. 5), we obtain

$$
\int_{0}^{h} \frac{\partial u_{c}}{\partial t} d z+\frac{u_{c}^{2}(h)-u_{c}^{2}(0)}{2}+\frac{P-P_{a}}{\rho}+g h=0,
$$

where $h(t)$ is the time-dependent height of water on the axis of the rocket, $P_{a}$ is atmospheric pressure, $u_{c}(h)$ is the (negative) vertical velocity of the water free surface on the axis of the rocket, and $u_{c}(0)$ is the exhaust velocity on the axis. Bernoulli's equation, as used in Refs. 2 and 3, results directly 
from Eq. (9) by neglecting the time derivative as well as gravity.

The order of magnitude of the first term in Eq. (9) is $H U / \tau$, which is equal to the magnitude of the second term $U^{2}$. As for the last two terms, using $P-P_{a}=10^{5} \mathrm{~Pa}, \rho$ $=1000 \mathrm{~kg} / \mathrm{m}^{3}, \quad H=0.1 \mathrm{~m}$, and apparent gravity $g$ $=1000 \mathrm{~m} / \mathrm{s}^{2}$, they are found to be comparable. Therefore, Eq. (9) cannot be simplified a priori to analyze the water rocket.

To use Eq. (9) for making predictions, assumptions have to be made about the flow pattern. We shall assume that the velocity profiles at various heights are self-similar, that is,

$$
u(z, r, t)=\frac{d h}{d t}\left[\frac{\Omega(h)}{\Omega(0)}\right] f\left(\frac{r}{R(z)}\right),
$$

where $R(z)$ is the radius of the bottle at height $z$ (see Fig. 5), $\Omega(z)=\pi R^{2}(z)$ is the cross sectional area at height $z$, and $f$ is a function that characterizes the velocity profile and satisfies $f(0)=1$. Uniform velocity is recovered with $f(x)=1$; the case where the water drops more rapidly at the center of the rocket corresponds to a function $f(x)$ with a maximum at $x$ $=0$. The ratio in the square brackets in Eq. (10) ensures that the total flow of water is the same over any section of the rocket. Moreover, the latter flow has to be equal to the rate of air expansion. Integrating Eq. (10) over a section leads to

$$
\frac{d V}{d t}=-\langle f\rangle \Omega(h) \frac{d h}{d t}
$$

with $\langle f\rangle=\int_{0}^{1} 2 x f(x) d x$.

By using Eq. (10), the various terms in Eq. (9) can be written explicitly as a function of $h(t)$, which leads to

$$
\begin{gathered}
I(h) \frac{d}{d t}\left(\Omega(h) \frac{d h}{d t}\right)+\frac{1}{2}\left(1-\left(\frac{\Omega(h)}{\Omega_{0}}\right)^{2}\right)\left(\frac{d h}{d t}\right)^{2} \\
+\frac{1}{\rho}\left(P_{i}\left(\frac{V_{i}}{V(t)}\right)^{\beta}-P_{a}\right)+g h=0
\end{gathered}
$$

with

$$
I(h)=\int_{0}^{h} \frac{d z}{\Omega(z)} .
$$

The notation highlights that this term accounts for the inertia of the accelerated water. In Eq. (12), $\beta$ is the polytropic exponent obtained in Sec. II A and $\Omega_{0}=\Omega(0)$ is the area of the nozzle.

If the rocket was forced to remain immobile, Eqs. (11) and (12) could be solved for $h(t)$ and $V(t)$. When the rocket is free to move, however, the gravity term $g$ includes a noninertial component resulting from the rocket's acceleration. The water ejection can, therefore, not be predicted independently of the rocket acceleration: the two problems are coupled and have to be solved simultaneously.

\section{Rocket acceleration}

In an inertial frame immobile with respect to the ground conservation of vertical momentum takes the form

$$
\frac{d}{d t} M U=\mathrm{Th}-W-D,
$$

where the total momentum of the rocket is written as $M U$, Th is the thrust, $W$ is the weight, and $D$ is the aerodynamic drag. We next discuss each of these terms.

For a conventional rocket, $M U$ is generally estimated by assuming that the fuel moves upward at the same speed as the rocket. This assumption does not hold for water rockets. More generally, the total momentum is

$$
M U=m \frac{d Z}{d t}+\int_{0}^{h} d z \int_{0}^{R(z)} 2 \pi r d r \rho u(z, r, t),
$$

where $m$ is the mass of the empty rocket, $Z$ is its vertical position, and $u(z, r, t)$ is the velocity of the water in the rocket. Note that, contrary to Sec. II B, the water velocity $u(z, r, t)$ is expressed here with respect to the ground. If we correct Eq. (10) by adding $d Z / d t$, Eq. (15) becomes

$$
M U=\left[m+\rho V_{w}\right] \frac{d Z}{d t}+\rho\langle f\rangle h \Omega(h) \frac{d h}{d t},
$$

where $V_{w}=\int_{0}^{h} \Omega(z) d z$ is the volume of water in the rocket. The second term in Eq. (16) is negative; it is specific to water rockets, and it was not taken into account in previous studies. ${ }^{2,3}$ This term accounts for the fact that the velocity of water in the rocket is smaller than the velocity of the rocket itself.

The three forces on the right-hand side of Eq. (14) are estimated in the usual way. The thrust is the rate of momentum transfer out of the rocket resulting from water expulsion. ${ }^{9}$ By using the velocity profile in Eq. (10), the thrust takes the form

$$
\mathrm{Th}=\rho \frac{\Omega(h)^{2}}{\Omega_{0}}\left(\frac{d h}{d t}\right)^{2}\left\langle f^{2}\right\rangle+\rho \Omega(h) \frac{d h}{d t} \frac{d Z}{d t}\langle f\rangle
$$

with $\left\langle f^{2}\right\rangle=\int_{0}^{1} 2 x f^{2}(x) d x$. For the case of a uniform velocity profile, the first contribution is the product of the rate of mass loss with the exhaust velocity; the second term accounts for the fact that the thrust is not estimated in the reference frame of the rocket. The last two forces exerted on the rocket are the weight and the aerodynamic drag. The weight is

$$
W=\left(m+\rho V_{w}\right) g_{0},
$$

where $g_{0}$ is the gravity, not to be confused with $g$ that enters Eq. (12) and includes a noninertial contribution. The aerodynamic drag is calculated in the usual way ${ }^{4,5}$ as

$$
D=C_{A} \Omega_{\max } \frac{\rho_{a}}{2}\left(\frac{d Z}{d t}\right)^{2},
$$

where $\rho_{a}$ is the density of air, $\Omega_{\max }$ is the maximum cross section area of the rocket, and $C_{A}$ is a dimensionless drag coefficient. For a rocket with a circular cross section and no side wings, $C_{A}$ is about $0.75,{ }^{4}$ but for the following analysis, it is sufficient to consider that its order of magnitude is 1 .

Combining Eqs. (14)-(19) leads to the following differential equation: 

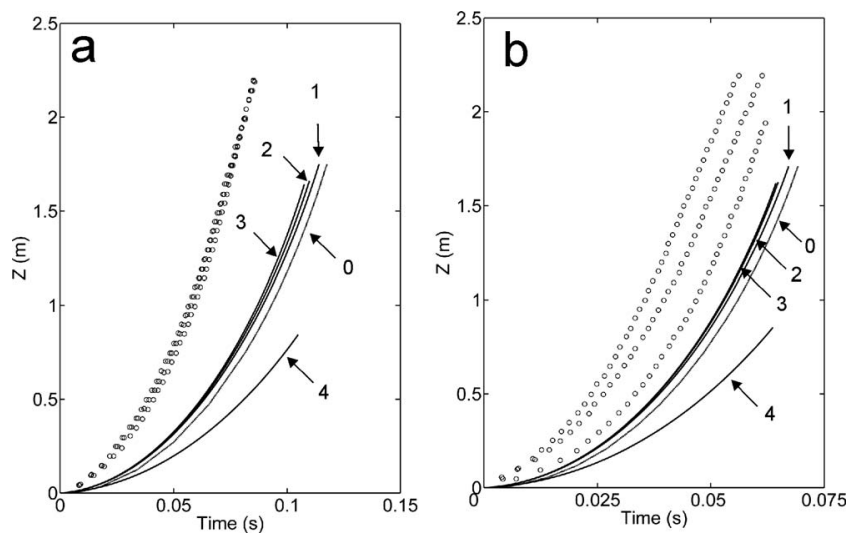

Fig. 6. Predicted and experimental heights reached by a water rocket as a function of time for (a) $P_{i}=3.4$ bars and (b) $P_{i}=6.8$ bars: $(\bigcirc)$ experimental data from Ref. 2, (0) textbook analysis, (1) current model with simplified geometry, (2) with realistic geometry, (3) with realistic geometry and vapor condensation, and (4) with realistic geometry, condensation, and nonuniform flow.

$$
\begin{aligned}
\frac{d}{d t}[(m & \left.\left.+\rho V_{w}\right) \frac{d Z}{d t}+\rho\langle f\rangle h \Omega(h) \frac{d h}{d t}\right] \\
= & \rho \frac{\Omega^{2}(h)}{\Omega_{0}}\left(\frac{d h}{d t}\right)^{2}\left\langle f^{2}\right\rangle+\rho \Omega(h) \frac{d h}{d t} \frac{d Z}{d t}\langle f\rangle \\
& -g_{0}\left(m+\rho V_{w}\right)-C_{A} \Omega_{\max } \frac{\rho_{a}}{2}\left(\frac{d Z}{d t}\right)^{2}
\end{aligned}
$$

which expresses the conservation of vertical momentum. Equations (11), (12), and (20) constitute a closed system of three differential equations that are coupled through the term $g=g_{0}+d^{2} Z / d t^{2}$ and have to be solved simultaneously for $Z(t), h(t)$, and $V(t)$.

\section{DISCUSSION}

\section{A. Numerical solutions and experimental data}

In this section the predictions of Eqs. (11), (12), and (20) are compared with the experimental data of Kagan et al. ${ }^{2}$ which are summarized in Fig. 6. These authors used a 2-1 soda bottle as a rocket, the takeoff of which they measured with a high-speed camera and a "smart-pulley" system. ${ }^{10}$ Their analysis was based on a dry adiabatic expansion, on Bernoulli's equation, and on the estimation of the thrust as the product mass loss rate and exhaust velocity. They also assumed that the rocket is a cylinder with a constant section area $\Omega$, ended by a sudden constriction to a smaller section $\Omega_{0}$. The predicted height as a function of time is reproduced in Fig. 6 as curve 0. As mentioned, the rocket outperforms this analysis.

The discrepancy between theory and experiment is not in contradiction with the findings of Finney, ${ }^{3}$ according to which the simplified analysis predicts the time of flight of the rocket with an accuracy better than $0.2 \mathrm{~s}$. Figure 6 shows that the velocity of the rocket is underestimated only at the beginning of the burnout; the velocity $v_{0}$ at the end of the burnout is predicted fairly accurately. As a consequence, the remaining time of flight after the end of the burnoutcalculated, for example, as $2 v_{0} / g$ when drag is neglected-is also accurately predicted. The main source of error for the total time of flight is the duration of the burnout, which is overestimated by less than $0.05 \mathrm{~s}$ for the case in Fig. 6(a). Accuracy better than $0.2 \mathrm{~s}$ is therefore needed to detect a discrepancy between theory and experiment on the basis of time of flight measurements.

We have accounted for several effects which were previously not considered and which may have an important role during the burnout. The first effect is water condensation during air expansion, which is accounted for by a polytropic exponent $\beta$ smaller than $\gamma$. The second effect is the rocket acceleration, which favors water ejection through increasing the apparent gravity in the rocket. From dimensional analysis, the acceleration is expected to be as significant as the pressure difference between the inside and the outside of the rocket. The third effect is the acceleration downward of the water inside the rocket, which reduces the vertical momentum of the rocket. On the other hand, water inertia is underestimated in the usual analysis, which makes use of Bernoulli's steady equation. The transient term, proportional to $I(h)$ in Eq. (12), is expected to reduce the overall rocket performance.

The procedure used for the numerical integration of Eqs. (11), (12), and (20) is discussed in Appendix A. We shall first assume the same rocket shape as in Ref. 2. For a perfect cylinder, we have $\Omega(h)=\Omega_{\max }$, and from Eq. (13), $I(h)$ $=h / \Omega_{\max }$. The numerical solutions for $P_{i}=3.4$ and 6.8 bars are plotted in Fig. 6 as curve 1 for $\beta=1.4$ (dry air) and $\langle f\rangle$ $=\left\langle f^{2}\right\rangle=1$ (a uniform velocity profile). For both pressures, curve 1 is slightly above the analysis of Ref. 2 , but the theoretical prediction remains below experiment.

A more realistic soda bottle has a transition from body to neck, as presented in Appendix B together with the corresponding values of $\Omega(h)$ and $I(h)$. The corresponding solutions of Eqs. (11), (12), and (20) are shown in Fig. 6 as curve 2. Another refinement of our analysis accounts for the condensation of water vapor using the exponents $\beta=1.31$ and $\beta=1.35$ as calculated in Sec. II A [see Fig. 3(b)]. The corresponding predictions are shown in Fig. 6 as curve 3. For the launch at $P_{i}=3.4$ bars, the performances are slightly improved by water vapor condensation, but the extra thrust is negligible for $P_{i}=6.8$ bars, as anticipated in Sec. II A.

The effect of a possible nonuniform velocity profile was also investigated. The velocity profile in Eq. (10) is modeled empirically as $f(x)=\exp \left(-0.5(x / \sigma)^{2}\right)$, which amounts to assuming that most of the flow occurs at the center of the rocket in a region that extends over a fraction $\sigma$ of its radius. With this form of $f(x)$ the coefficients $\langle f\rangle$ and $\left\langle f^{2}\right\rangle$ are $\langle f\rangle$ $=2 \sigma^{2}\left(1-\exp \left(-0.5 / \sigma^{2}\right)\right)$ and $\left\langle f^{2}\right\rangle=\sigma^{2}\left(1-\exp \left(-1 / \sigma^{2}\right)\right)$. With these coefficients, we found that any finite value of $\sigma$ results in lower performances of the rocket, as exemplified in the figure for $\sigma=0.5$ (curve 4).

\section{B. Analysis of simplified equations}

Although some of the effects included in the present analysis bring theory slightly closer to experiment, the improvements are small: a real rocket still outperforms the theoretical prediction. To try and understand why, we now consider the simplified cylindrical model with $\Omega(h)=\Omega_{\max }$ for $h>0$ and $I(h)=h / \Omega_{\max }$. It is shown in Appendix A that gravity and aerodynamic drag are negligible during the thrust 

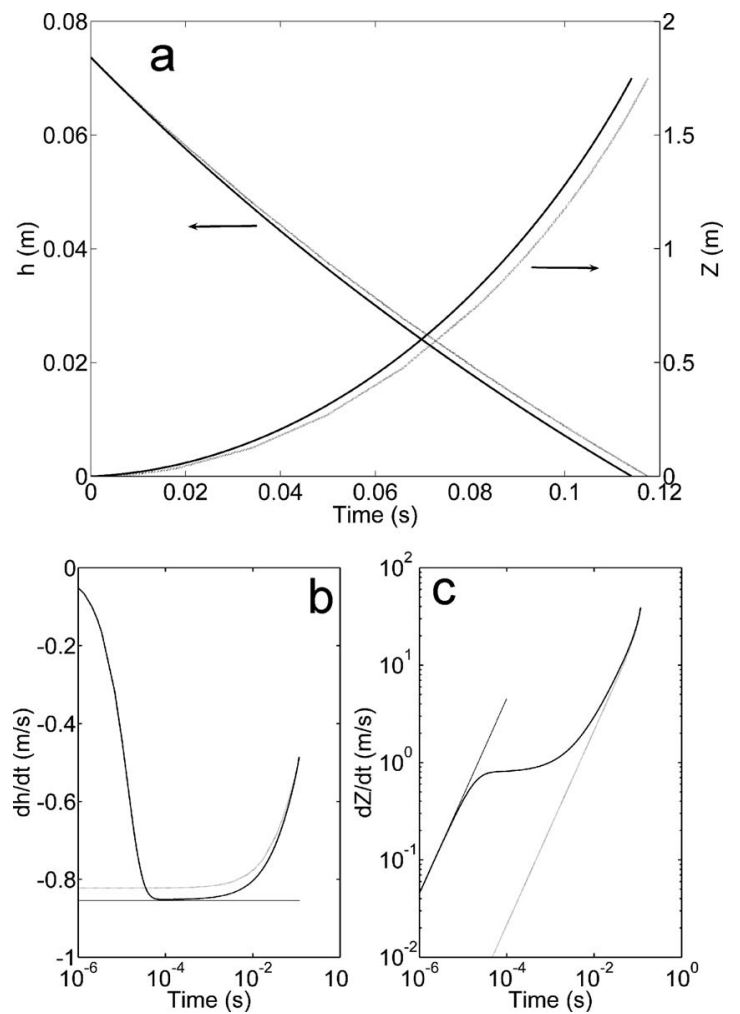

Fig. 7. Water height $h$ and altitude $Z$ of the rocket according to textbook (gray) and the present (black) analyses using conditions from Ref. 2 with $P_{i}=3.4$ bars. In (b) and (c) the velocities are plotted on a logarithmic time scale to stretch the shorter times. The horizontal line in (b) is given by Eq (24), and the initial slope in (c) is $d^{2} Z / d t^{2} \approx(\Omega \Delta P) / m$.

phase, and thus we shall here assume $g_{0}=0$ and $C_{A}=0$. With these assumptions, Eqs. (12) and (20) take the form

$$
h \frac{d^{2}}{d t^{2}}(h+Z)=-\frac{\Delta P}{\rho}+\frac{1}{2}\left[\left(\frac{\Omega}{\Omega_{0}}\right)^{2}-1\right]\left(\frac{d h}{d t}\right)^{2}
$$

and

$$
\frac{m}{\rho \Omega} \frac{d^{2} Z}{d t^{2}}+h \frac{d^{2}}{d t^{2}}(h+Z)=\left[\left(\frac{\Omega}{\Omega_{0}}\right)-1\right]\left(\frac{d h}{d t}\right)^{2},
$$

where $\Delta P$ is the time-dependent pressure difference between the inside and the outside of the rocket.

The solution of Eqs. (21) and (22) for $P_{i}=3.4$ bars is shown in Fig. 7(a), together with the solution of the simplified equations of Ref. 2. The time derivatives $d h / d t$ and $d Z / d t$ are plotted in Figs. 7(b) and 7(c) on a logarithmic time axis to stretch the short times. The curves exhibit three distinct time intervals. For very short times $\left(t<10^{-4} \mathrm{~s}\right)$, the rocket accelerates uniformly [Fig. 7(c)]. This interval is followed by one $\left(10^{-4} \mathrm{~s}<t<10^{-2} \mathrm{~s}\right)$ during which the rate of water ejection is maximum [Fig. 7(b)], and the vertical speed of the rocket $d Z / d t$ is a constant [plateau in Fig. 7(c)]. For $t>10^{-2} \mathrm{~s}$, the rate of water ejection decreases monotonically and the rocket accelerates again. The latter stage is the only visible one on the time scale of Fig. 7(a).
To analyze the first two intervals, we eliminate the second time derivative of $(h+Z)$ between Eqs. (21) and (22), leading to

$$
\frac{m}{\rho \Omega} \frac{d^{2} Z}{d t^{2}}+\frac{1}{2}\left[\frac{\Omega}{\Omega_{0}}-1\right]^{2}\left(\frac{d h}{d t}\right)^{2}=\frac{\Delta P}{\rho} .
$$

The initial value of $d h / d t$ is 0 , and thus the middle term in Eq. (23) is negligible at the onset of water ejection. The initial vertical acceleration of the rocket is found to be $d^{2} Z / d t^{2} \approx(\Omega \Delta P) / m$, in excellent agreement with the numerical solution [initial slope in Fig. $7(\mathrm{c})]$. Note that $\Delta P$ can be considered to be a constant over a time scale as short as in Figs. 7(b) and 7(c). Because the maximum in $d h / d t$ is reached for $d^{2} Z / d t^{2}=0$, the maximum rate of water ejection is obtained by neglecting the first term in Eq. (23), which leads to

$$
\frac{d h}{d t} \approx-\frac{\sqrt{2 \Delta P / \rho}}{\Omega / \Omega_{0}-1},
$$

which is plotted as a horizontal line in Fig. 7(b), in agreement with the numerical solution. Incidentally, the level of the plateau in Fig. $7(\mathrm{c})$ is found to be $-d h / d t$, which means that the height of the water surface is initially a constant with respect to the ground.

The maximum rate of water ejection predicted by Eq. (24) is slightly larger than that predicted by Bernoulli's equation, as is seen in Fig. 7(b). Bernoulli's ejection rate is obtained by neglecting the time derivative in Eq. (21), which amounts to replacing the factor $\Omega / \Omega_{0}-1$ in Eq. (24) by $\sqrt{\left(\Omega / \Omega_{0}\right)^{2}-1}$. For large values of $\Omega / \Omega_{0}$, the two numbers are very close to each other. In general, applying Bernoulli's equation leads to an underestimation of the initial ejection velocity. Over longer times, the evolution of $h(t)$ and $Z(t)$ predicted by Eqs. (23) and (24) and by the simplified model ${ }^{2}$ are similar [Fig. 7(a)].

\section{CONCLUSIONS AND PROSPECTS}

This paper was motivated by the observation ${ }^{2}$ that water rockets outperform the usual textbook analysis at the beginning of the thrust phase. The present analysis takes account of several phenomena that were previously not considered. These corrections bring theory slightly closer to experiment, but a discrepancy persists that is larger than the experimental uncertainty.

The present analysis does answer some questions raised in Ref. 2. Should the energy released by the condensation of water be included in the theory? If so, how? We have shown that this energy can be accounted for using a polytropic exponent smaller than $\gamma=1.4$. The value of the exponent has to be calculated from the initial pressure and temperature. Notably, the performance of a rocket can be increased if it is filled with hot water. Advanced users may also want to try other liquids. As a rule of thumb, the liquid should be very volatile with a large enthalpy of evaporation. Why is the size of the hole in the bottle so crucial? The movement of water inside the rocket contributes significantly to the total momentum of the rocket, an effect that is more pronounced for a larger hole. This effect is absent in other types of rockets. 
Incidentally, the movement of water within the rocket is also expected to stabilize it, in agreement with an observation reported in Ref. 2.

We also found that any deviation from a uniform water velocity profile over the rocket section is detrimental to the performance of the rocket. The shape of the rocket also influences water ejection in a way that can be calculated numerically through the function $I(h)$, which offers the prospect of finding the optimal rocket's shape. In this respect, current water rockets are energetically very inefficient. Maximum energy efficiency would require that water be ejected with a constant velocity with respect to the ground. ${ }^{11}$ As noted when discussing Fig. 2 some of the ejected water moves upward, which leaves much room for optimization.

These partial results leave the main question more relevant than ever: What is the origin of the extra thrust at the beginning of the burnout? Should the elastic deformation of the rocket be included in the analysis? Should the interaction of the water jet with the ground be taken into account? The water rocket has long been used to teach momentum conservation, elementary fluid mechanics, and thermodynamics. There is more to learn from it: for instance, that some simple and popular devices are still lacking a satisfactory physical analysis.

\section{ACKNOWLEDGMENTS}

The author is a postdoctoral researcher of the National Funds for Scientific Research (F.R.S.-FNRS, Belgium). He is indebted to Professor Georges Heyen (University of Liège) for checking the numerical accuracy of the analysis in Sec. II A and to Dario Zilli (Universidad de Buenos Aires, Argentina) and Sebastian Cativa-Tolosa (Comision Nacional de Energia Atomica, Argentina) for sharing their enthusiasm about water rockets during their stay in Belgium.

\section{APPENDIX A: DIMENSIONLESS FORM OF THE GOVERNING EQUATIONS AND THEIR NUMERICAL INTEGRATION}

To estimate the order of magnitude of the different terms in the governing equations, and to make their numerical integration easier, the equations derived in Sec. II are here expressed in dimensionless form. The height of the rocket $H$ is used as the length unit, with notation $\bar{h}=h / H$ and $\bar{Z}$ $=z / H$. For the time unit, we choose $\tau=\sqrt{\rho H^{2} / P_{a}}$, which is the order of magnitude of the time needed for a pressure difference of $P_{a}$ to accelerate an unrestricted column of fluid of density $\rho$ and height $H$, starting from rest, over a distance $H$. The dimensionless time is $\bar{t}=t / \tau$.

Using these dimensionless variables, Eqs. (11), (12), and (20) can be put into the form

$$
\begin{aligned}
& \frac{d \bar{V}}{d \bar{t}}=-\langle f\rangle \omega(\bar{h}) \frac{d \bar{h}}{d \bar{t}}, \\
& \bar{I}(\bar{h}) \frac{d}{d \bar{t}}\left(\bar{\omega}(\bar{h}) \frac{d \bar{h}}{d \bar{t}}\right)+\frac{1}{2}\left(1-\left(\frac{\omega(\bar{h})}{\omega_{0}}\right)^{2}\right)\left(\frac{d \bar{h}}{d \bar{t}}\right)^{2} \\
& +\left(\bar{P}_{i}\left(\frac{V_{i}}{V}\right)^{\beta}-1\right)+\left(\Gamma_{0}+\frac{d^{2} \bar{Z}}{d \bar{t}^{2}}\right) \bar{h}=0, \\
& \frac{d}{d t}\left[\left(\bar{m}+\bar{V}_{w}\right) \frac{d \bar{Z}}{d \bar{t}}+\langle f\rangle \bar{h} \omega(\bar{h}) \frac{d \bar{h}}{d \bar{t}}\right] \\
& =\frac{\omega^{2}(\bar{h})}{\omega_{0}}\left(\frac{d \bar{h}}{d \bar{t}}\right)^{2}\left\langle f^{2}\right\rangle+\omega(\bar{h}) \frac{d \bar{h}}{d \bar{t}} \frac{d \bar{Z}}{d \bar{t}}\langle f\rangle \\
& -\Gamma_{0}\left(\bar{m}+\bar{V}_{w}\right)-\frac{\rho_{a}}{\rho} \frac{1}{2} C_{A}\left(\frac{d \bar{Z}}{d \bar{t}}\right)^{2}
\end{aligned}
$$

with $\omega=\Omega / \Omega_{\max }, \omega_{0}=\Omega_{0} / \Omega_{\max }, \bar{P}=P / P_{a}, \bar{I}=\Omega_{\max } I / H, \Gamma_{0}$ $=\rho g_{0} H / P_{a}, \quad \bar{V}_{w}=V_{w} /\left(\Omega_{\max } H\right), \quad \bar{m}=m /\left(\rho V_{w}\right), \quad$ and $\bar{V}$ $=V /\left(\Omega_{\max } H\right)$.

The choice of the time unit $\tau$ makes all time derivatives of the order of 1 in Eqs. (A1)-(A3). For $H=0.2 \mathrm{~m}$, we find $\Gamma_{0}=0.02$, and hence gravity is negligible during the water ejection. The magnitude of the aerodynamic drag in Eq. (A3) is $\rho_{a} / \rho=0.001$, which means that the drag is also negligible during this phase.

The numerical integration of Eqs. (A1)-(A3) requires that they be written as a system of first-order differential equations. For that purpose, we introduce the variables

$$
p_{h}=\omega(\bar{h}) \frac{d \bar{h}}{d \bar{t}}
$$

and

$$
p_{Z}=\frac{d \bar{Z}}{d \bar{t}}
$$

which enable us to write Eqs. (A1)-(A3) in matrix form

$$
\left(\begin{array}{ccccc}
1 & 0 & 0 & 0 & 0 \\
0 & 1 & 0 & 0 & 0 \\
0 & 0 & \bar{I}(\bar{h}) & 0 & \bar{h} \\
0 & 0 & 0 & 1 & 0 \\
-p_{h} & 0 & \langle f\rangle \bar{h} & 0 & \left(\bar{m}+\bar{V}_{r}-\bar{V}\right)
\end{array}\right) \frac{d}{d \bar{t}}\left(\begin{array}{c}
\bar{V} \\
\bar{h} \\
p_{h} \\
\bar{Z} \\
p_{Z}
\end{array}\right)=\left(\begin{array}{c}
-\langle f\rangle p_{h} \\
p_{h} / \omega \\
\left(\left\langle f^{2}\right\rangle / \omega_{0}^{2}-\langle f\rangle / \omega^{2}\right) p_{h}^{2}+\langle f\rangle p_{h} p_{Z}-\Gamma_{0}\left(\bar{m}+\bar{V}_{r}-\bar{V}\right)-0.5\left(\rho_{a} / \rho\right) C_{A} p_{Z}^{2}
\end{array}\right),
$$




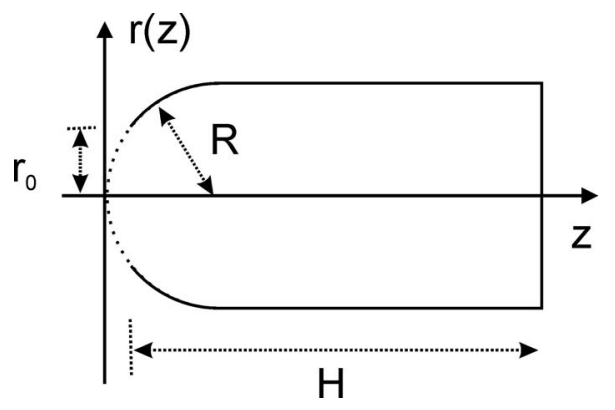

Fig. 8. A more realistic model of a soda bottle, consisting of a cylinder of radius $R$ ended by a hemispherical cap truncated at the bottle neck. The radius $r_{0}$ of the neck has been exaggerated for clarity.

where we have replaced $\bar{V}_{w}$ by $\bar{V}_{r}-\bar{V}$, and $\bar{V}_{r}=V_{r} /\left(\Omega_{\max } H\right)$ is the dimensionless total volume of the rocket. By using the form of Eq. (A6), the governing equations of the water rocket can be easily solved numerically.

\section{APPENDIX B: A REALISTIC SODA BOTTLE SHAPE}

We assume here that the soda bottle of Ref. 2 is a cylinder of radius $R$ with a hemispherical cap truncated at the bottle neck (Fig. 8). If we let $R_{0}$ be the radius of the neck, the shape of the bottle is given by

$$
r(z)=\left\{\begin{array}{cl}
R \sqrt{z / R(2-z / R)} & \text { for } z_{0} \leq z \leq R \\
R & \text { for } R<z \leq H+z_{0},
\end{array}\right.
$$

where $z_{0}=R-\sqrt{R^{2}-R_{0}^{2}}$ is the position of the neck, and $H$ is the total height of the bottle. Using Eq. (B1), the dimensionless section of the rocket is

$$
\omega=\frac{\bar{z}_{0}+\bar{h}}{\bar{R}}\left[2-\frac{\bar{z}_{0}+\bar{h}}{\bar{R}}\right] \text { for } \bar{h} \leq \bar{R}-\bar{z}_{0}
$$

and $\omega=1$ for larger values of $\bar{h}$. From Eq. (13), we find

$\bar{I}= \begin{cases}\frac{\bar{R}}{2} \ln \left(\frac{\left(\bar{z}_{0}+\bar{h}\right)\left(2 \bar{R}-\bar{z}_{0}\right)}{\bar{z}_{0}\left(2 \bar{R}-\bar{z}_{0}-\bar{h}\right)}\right) & \text { for } \bar{h} \leq R-\bar{z}_{0} \\ \bar{R} \ln \left(\frac{\left(2 \bar{R}-\bar{z}_{0}\right)}{\bar{z}_{0}}\right)+\bar{h}-\left(\bar{R}-\bar{z}_{0}\right) & \text { otherwise, }\end{cases}$

which function is needed to predict the rate of water ejection through Eq. (12).

\footnotetext{
${ }^{1}$ See, for example, the article on water rockets in 〈en.wikipedia.org/wiki/ Water_rocket) and links therein.

${ }^{2}$ D. Kagan, L. Buchholtz, and L. Klein, "Soda-bottle water rockets," Phys. Teach. 33, 150-157 (1995).

${ }^{3}$ G. A. Finney, "Analysis of a water-propelled rocket: A problem in honors physics," Am. J. Phys. 68, 223-227 (2000).

${ }^{4}$ L. D. Landau and E. Lifshitz, Fluid Mechanics (Pergamon, Oxford, 1987).

${ }^{5}$ R. Bird, W. Stewart, and E. Lightfoot, Transport Phenomena (Wiley, New York, 1962).

${ }^{6}$ I. Prigogine and D. Kondepudi, Modern Thermodynamics: From Heat Engines to Dissipative Structures (Wiley, New York, 1998).

${ }^{7}$ R. P. Feynman, The Feynman Lectures on Physics (Addison-Wesley, Reading, MA, 1970).

${ }^{8}$ G. F. C. Rogers and Y. Mayhew, Engineering Thermodynamics: Work and Heat Transfer (Longman, Singapore, 1992).

${ }^{9}$ R. A. Serway and J. Jewett, Physics for Scientists and Engineers, 6th ed. (Brooks-Cole, Belmont, CA, 2003).

${ }^{10}$ R. A. Jenkins, "Measuring model rocket acceleration," Phys. Teach. 31, 10-15 (1993).

${ }^{11}$ R. H. Gowdy, "The physics of perfect rockets," Am. J. Phys. 63, 229232 (1995).
}

\section{ALL BACK ISSUES ARE AVAILABLE ONLINE}

The contents of the American Journal of Physics are available online. AJP subscribers can search and view full text of AJP issues from the first issue published in 1933 to the present. Browsing abstracts and tables of contents of online issues and the searching of titles, abstracts, etc. is unrestricted. For access to the online version of AJP, please visit http://aapt.org/ajp.

Institutional and library ("nonmember") subscribers have access via IP addresses to the full text of articles that are online; to activate access, these subscribers should contact AIP, Circulation \& Fulfillment Division, 800-344-6902; outside North America 516-576-2270 or subs@aip.org.

Individual ("member") subscribers to the print version who wish (for an additional fee) to add access to the online version should contact AAPT or go to the AAPT website: https://www.aapt.org/Membership/secure/agreement.cfm. 